\title{
Rapid volumetric optoacoustic imaging of neural dynamics across the mouse brain
}

\author{
Sven Gottschalk ${ }^{1}$, Oleksiy Degtyaruk ${ }^{1,2}$, Benedict Mc Larney ${ }^{1,2}$, Johannes Rebling ${ }^{1,2}$, \\ Magdalena Anastasia Hutter ${ }^{1,3}$, Xosé Luís Deán-Ben ${ }^{1}$, Shy Shoham ${ }^{4}{ }^{*}$, Daniel Razansky ${ }^{1,2,{ }^{*}}$ \\ ${ }^{1}$ Institute for Biological and Medical Imaging, Helmholtz Center Munich, Neuherberg, Germany \\ ${ }^{2}$ Faculty of Medicine, Technical University of Munich, Germany \\ ${ }^{3}$ Department of Electrical and Computer Engineering, Technical University of Munich, Germany \\ ${ }^{4}$ Neuroscience Institute, New York University Langone Medical Center, New York, USA
}

\section{Abstract}

\begin{abstract}
Ongoing efforts to scale neuroimaging towards direct visualization of mammalian brain-wide neural activity face major challenges, and a large gap still exists between localized optical microscopy looking at rapid cellular-resolved neuronal activities and whole-brain observations of slow hemodynamics and metabolism provided by macroscopic imaging modalities. Optoacoustic imaging holds inherent advantages for deep tissue observations, but to date has not been applied towards direct activity observation in the mammalian brain. Here we demonstrate in vitro and in vivo functional optoacoustic neuroimaging from mice expressing the genetically encoded calcium indicators GCaMP6, effectively bridging the gap between functional microscopy and whole-brain macroscopic neuroimaging. We yielded instantaneous high-resolution 3D snapshots of wholebrain activity maps with single optoacoustic excitations and enabled non-invasive detection of fast neural responses to sensory stimuli in the presence of strong hemoglobin background absorption. These results demonstrate a new enabling technique towards scalable direct neuroimaging at unprecedented penetration depths and spatio-temporal resolutions.
\end{abstract}

\begin{abstract}
Mammalian brain function arises through multi-scale interactions in a highlyinterconnected, complex network of cortical and sub-cortical neurons ${ }^{1}$. Its understanding relies heavily upon experimental methods for monitoring neuronal activity at different spatial and temporal scales ${ }^{2}$. Whole-brain activity is typically imaged in both humans and small animals using methods that only indirectly reflect neural activity ${ }^{3-5}$, and thus recent efforts towards directly mapping neuronal network function have focused on fluorescence microscopy and the emerging toolbox of calcium and voltage indicators ${ }^{6}$. Techniques like two-photon microscopy can visualize the activity of thousands of neurons across multiple cortical layers ${ }^{7}$. However, optical brain microscopy approaches generally require invasivity, namely, skull and/or scalp removal, and are further unable to resolve large-scale neural activity across entire scattering rodent brains due to intense photon scattering. Despite recent developments in fluorescence microscopy ${ }^{8-10}$, achieving imaging rates exceeding $1 \mathrm{~mm}^{3} / \mathrm{s}$ is
\end{abstract}

\footnotetext{
*Corresponding authors: shoham@nyu.edu, dr@tum.de.
} 
still challenging ${ }^{2,10}$ and real-time direct monitoring of large-scale neuronal activity in the mouse brain remains an unmet need.

Hybrid opto-acoustic (OA) tomography techniques are ideally suited for real-time volumetric brain interrogations due to their intrinsic resilience to light scattering with a single laser pulse sufficient for inducing OA responses in the entire imaged tissue volume ${ }^{11}$. This provides an optimal trade-off between volumetric imaging rates and spatial resolution not achievable with other neuroimaging modalities ${ }^{11-13}$. Broadband ultrasound waves are generated by transient light absorption, enabling high-resolution imaging of optical contrast at centimeter-scale depths in tissue ${ }^{14,15}$, an order of magnitude gain over state-of-the-art optical microscopy. In addition, hemoglobin manifests excellent endogenous absorption contrast, and OA naturally attains label-free high fidelity images of vascular anatomy, blood oxygenation level and their respective dynamics, enabling the assessment and monitoring of multiple activity-related hemodynamic parameters in health and disease $\mathrm{e}^{12,13,15-20}$.

Can the benefits of OA imaging be harnessed towards direct neuronal activity imaging? In recent work we demonstrated robust calcium-dependent OA contrast using a genetically encoded calcium indicator (GECI) in zebrafish larvae and excised brains ${ }^{11}$. While promising, the initial proof-of-concept demonstrations left important questions unanswered, ranging from the basic ability to image calcium responses in large and highly scattering and absorbing mammalian brains to whether the method is sensitive enough to observe natural sensory responses relative to the high background hemoglobin absorption signals at GCaMP excitation wavelengths. Here we devised customized functional OA neuro-tomography setups in order to demonstrate and characterize the capacity for real-time volumetric imaging of calcium transients across mouse brains expressing GECIs, further providing a first demonstration that the OA method is sufficiently sensitive for detection of distributed sensory responses and setting the stage for future advances.

\section{RESULTS}

\section{An isolated brain preparation}

To study the fundamental ability to image whole-brain calcium dynamics in mammalian brain optoacoustically, we first developed and validated an isolated-brain preparation from GCaMP6f-expressing mice (see Methods for details of the model) and a custom imaging setup continuously perfused with artificial cerebrospinal fluid (ACSF). The blood-free model eliminates background hemodynamic signals, thus allowing for an unequivocal initial characterization of the calcium-related optoacoustic signal variations. It further enables deeper penetration of the $488 \mathrm{~nm}$ light used for the GCaMP excitation. The isolated brain model exhibits high viability and functional neuronal activity for several hours (Suppl. Figs. 1-3) and realistic optical light scattering and indicator responses. To evaluate neuronal viability of the in vitro model we intracortically injected isolated brains of CD-1 mice ( $n=3)$ with $10 \mathrm{kD}$ dextran-conjugated Texas Red, an anterograde/retrograde tracer transported by live neurons ${ }^{21}$. The tracer stained structures up to $2 \mathrm{~mm}$ away from the injection site, with distant staining observed exclusively in the viable dextran-conjugated dye group (Suppl. Figs. 1C-E). We further recorded electroencephalography (EEG) signals from the cortices of isolated brains perfused in oxygenated $\operatorname{ACSF}(n=3)$ vs. non-vital controls $(n=3)$ and in vivo 
recordings $(n=3)$ in order to evaluate the preparation's electrophysiological function. We observed that injection of the epileptic drug pentylenetetrazol (PTZ) caused significant signal amplitude increases in EEG recordings, particularly in the higher 10-20 Hz frequency range in both isolated and in vivo brains, but not in the non-vital control brains (Suppl. Fig. 3), nor following injection of a 10-fold lower PTZ concentration ${ }^{22}$ (Fig. 3B).

\section{Volumetric optoacoustic and planar fluorescence of calcium activity in whole isolated mouse brains}

The developed hybrid imaging system allows for volumetric OA monitoring of resting and stimulus-evoked calcium dynamics concurrently with planar fluorescence imaging (Fig. 1). In the experimental set-up, seven optical fibers coupled to a pulsed laser source equally illuminated the brain from multiple directions while planar fluorescence and volumetric OA images were simultaneously captured using a sensitive camera and spherical-matrix ultrasound detection array (see schematic in Fig. 1A and Methods for details). The 512element spherical matrix transducer array with $5 \mathrm{MHz}$ central frequency and $140^{\circ}$ angular tomographic coverage (Fig. 1B) was specifically designed to provide a field-of-view (FOV) of $\sim 2 \mathrm{~cm}^{3}$ effectively covering the entire mouse brain with nearly isotropic three-dimensional (3D) resolution of $\sim 150 \mu \mathrm{m}$ (see characterization data in Suppl. Fig. 4A). This corresponds to about one million individual voxels that can be visualized within the FOV at a volumetric imaging rate of $100 \mathrm{fps}$ (the maximal pulse repetition rate of the excitation laser). The system affords the acquisition of volumetric OA images covering the entire GCaMP6fexpressing brain along the transverse, sagittal and coronal planes with high resolution (Figs. $1 \mathrm{C}$ and $1 \mathrm{E} ; \mathrm{n}=4$ brains imaged), providing a stark qualitative improvement over diffuse, surface-weighted 2D wide-field fluorescence image lacking the depth resolution (Fig. 1D). A range of gross anatomical features can be identified in the excised brains, such as the cortices, cerebellum, medulla, thalamus, and hippocampus (Figs. 1E and 1F), demonstrating the system's ability to volumetrically capture information from the entire brain.

OA measurements of purified GCaMP6f proteins (Fig. 2A) rendered spectrally specific calcium-dependent absorption changes, suggesting that calcium concentration changes should be optoacoustically detectable in addition to their fluorescence signature. We therefore used the new system to acquire activity videos in isolated GCaMP6f mouse brains resulting from frontal-cortical injection of PTZ $(n=4)$. This neuro-activating agent is assumed to interfere with GABAergic signaling and actuates fast seizure-like activity in the nervous system ${ }^{23}$. Large OA responses of up to $150 \%$ were readily observed following the injection (Fig. 2B), appearing to spatially propagate around the injection site (see the location of glass capillary in Fig. 1D) and into the uninjected hemisphere (Figs. 2E and 2F). This is consistent with previous observations of PTZ-induced brain-wide propagating calcium waves ${ }^{24}$, putatively indicating the preservation of inter-hemispheric communication in this in vitro model. Similar changes were also observed in the simultaneously acquired wide-field planar fluorescence images (Suppl. Figs. 5A,C), independently validating that the detected OA signals directly correspond to neural calcium dynamics. These responses were abolished during concurrent injections of the sodium channel blocker tetrodotoxin (TTX), a potent suppressor of neural activity (Fig. 2B). PTZ injection into control CD-1 mouse brains expressing no GCaMP6f resulted in no detectable OA or fluorescence signal increases $(n=3$, 
Fig. 2C and Suppl. Figs. 5B,D), and neither did control PBS injections into GCaMP6flabeled brains (n=3, Fig. 2D and Suppl. Fig. 5E). Notably, the observed relative fluorescence changes were an order of magnitude smaller (only up to $30 \%$ above baseline), most likely due to the diffuse nature and higher background noise of the wide-field fluorescence modality.

\section{Non-invasive 3D optoacoustic imaging of the whole mouse brain in vivo}

We next explored non-invasive (through intact skin and skull) volumetric in vivo OA recordings from the whole mouse brain using a customized experimental arrangement. Here, the ultrasound array's orientation was reversed and fluorescence versus OA imaging were performed sequentially due to geometrical constraints of the in vivo measurement setup (Fig. $3 \mathrm{~A}$ and Suppl. Fig. 6). GCaMP6f expression was clearly visible in the somatosensory cortex of the transgenic mice in vivo (Fig. 3B). The 3D OA images mainly exhibit vascular contrast related to the strong light absorption by hemoglobin (Fig. 3C). In addition, the limited light penetration at the peak excitation wavelength of GCaMP6f $(488 \mathrm{~nm})$ is evident: at $488 \mathrm{~nm}$ clear contrast is preserved up to $\sim 1.5 \mathrm{~mm}$ in depth (Fig. 3C), while images at the $650 \mathrm{~nm}$ wavelength, where blood absorption diminishes significantly, clearly show visible absorption contrast across the entire brain down to $\sim 7 \mathrm{~mm}$ depth (Fig. 3D). The fluorescence signal photo-bleaches at a moderate rate of $\sim 5 \%$ signal loss per 100 seconds of imaging (Fig. 3E, left) under experimental illumination conditions $\left(\sim 3 \mathrm{~mJ} / \mathrm{cm}^{2}\right.$ excitation light fluence reaching the scalp surface). This rate is consistent with the bleaching rate of the OA signal (Fig. 3E, right) and with earlier observations ${ }^{25}$. The fluorescence also recovered to pre-imaging levels within 24 hours due to protein recycling (data not shown). In order to further minimize protein bleaching, the laser beam could potentially be blocked for time intervals when no activity is expected.

\section{Brain-wide optoacoustic imaging of stimulus-locked calcium responses in vivo}

We next used the in vivo imaging set-up to explore volumetric recording of somatosensoryevoked rapid calcium transients in GCaMP6f/6s-expressing mice. To detect stimulus-locked fluorescence and OA calcium-signals (Figs. 4A-B) while minimizing background hemodynamic responses ${ }^{26}$, brief $50 \mathrm{~ms}$ electrical stimulation pulses were repeatedly delivered to the hind-paw every 20s (Fig. 4C). Clearly resolvable stimulus-locked OA responses to each stimulus were readily observed in GCaMP6f mice ( $n=4$ experiments in 3 mice), with typical average traces reaching a peak at $\sim 360 \mathrm{~ms}$ post stimulation, then rapidly decaying and reaching the baseline level at $\sim 0.8$ s post stimulation (Figs. $4 \mathrm{~B}-\mathrm{C}$ ), paralleling the temporal patterns of the observed fluorescence responses acquired at a lower $6.25 \mathrm{fps}$ temporal resolution (Figs. 4A, C). Interestingly, the normalized OA signal transients $(\triangle \mathrm{OA} / \mathrm{OA})$ in the activated brain area were spatially correlated with and generally stronger than the separately measured normalized $\Delta \mathrm{F} / \mathrm{F}$ fluorescence responses (Fig. 4D), despite the very high blood background absorption levels inherently reflected in the OA signals. This difference is presumably attributable to intrinsic differences between these modalities, where the fluorescence signal traces effectively average calcium responses from a larger volume such differences can be particularly prominent at deep locations, as also observed in the isolated brain experiments where OA and fluorescence responses were measured concurrently. Also the in vivo OA recordings from deeper slices (Fig. 4B) exhibit distinct 
activation patterns not resolvable in the surface-weighted mesoscopic fluorescence images affected by the strong light scattering in the scalp, skull and the brain. Thus, OA provides the additional depth information without sacrificing speed, allowing it to resolve neuronal activity in 3D with unparalleled spatio-temporal resolution (Suppl. Figs. 4D-F). Note that signal averaging across repeated responses results in a better rejection of noise and a clearer distinction of the activation peaks (Fig. 4E-F). No OA signal changes were detected in wildtype mice under the same brief 50ms electrical stimulation paradigm (Fig. 4G), confirming that this particular paradigm does not induce detectable hemodynamic changes.

We further analyzed the functional OA signals of different voxels located in the somatosensory cortex and a major vessel (superior sagittal sinus, SSS). Clear bilateral activations can be seen in both planar fluorescence (Fig. 4E) and OA traces recorded from the somatosensory cortex areas outside major vessels at approximately $1 \mathrm{~mm}$ depths (Fig. 4F), and also in the stimulus-locked 4D spatio-temporal OA image sequences (Fig. 4B), which is consistent with previous reports of bilateral somatosensory cortical activations ${ }^{27,28}$. Bilateral activations were also consistently observed in control experiments using different strengths of the stimulation pulse, a different breathing gas and after using analgesia prior to stimulation experiments (Suppl. Fig. 7). To further corroborate that the detected signals correspond to calcium transients rather than to hemodynamic changes, we imaged GCaMPnegative (wild-type) mice as controls using the same stimulation paradigm, observing no signal spikes (Fig. 4G). On the other hand, when changing the stimulation paradigm to a longer stimulation pulse train, known to induce robust bilateral hemodynamic responses in BOLD MRI ${ }^{29}$, OA signal changes could be clearly observed using multi-spectral measurements (Supp. Fig. 8). These findings show that both fast calcium-related activity and slow hemodynamic responses can be effectively measured optoacoustically.

We also compared the OA responses of mice expressing fast GCaMP6f and slow GCaMP6s protein variants (Fig. 5A) and subjected to the same hind-paw stimulation paradigm. Similar brain activation patterns and relative OA signal increases were observed for both mouse strains (cortical layers at $\sim 1 \mathrm{~mm}$ depth shown in Fig. 5B). The peak responses for GCaMP6f and GCaMP6s signals were produced at approximately the same time point following the stimulation pulse, while a slower decay was clearly observed in the GCaMP6s signals (Figs. 5C, left panel, and 5D, left panel). These observations are consistent with the time constants measured in fluorescence measurements (Figs. 5C, right panel, and 5D, right panel, images not shown) and with previously reported protein dynamics ${ }^{30}$.

\section{OUTLOOK}

Extensive efforts in optical neuroimaging are directed towards increasing the effective field of view and volumetric imaging rates of functional microscopy techniques, but the inherent advantages of OA approaches may allow the mapping of brain activity at depths and spatiotemporal scales not achievable by other modalities. Our study is the first to examine fast OA signatures of GECIs in the densely vascularized and light-scattering mammalian brain, further showing that changes in their fluorescence are directly related to the OA responses, both in vitro and in vivo. We found that despite strong background hemoglobin absorption at the effective imaging wavelengths of GCaMP-type calcium indicators, the OA modality is 
sensitive enough to record sensory-evoked brain activity via GCaMP6f and GCaMP6s calcium-related signal changes that were in fact stronger than the corresponding fluorescence responses. This represents an important milestone for direct OA neuroimaging, which is further empowered by its deep tissue real-time 3D imaging performance and the capacity to sense multiple cerebral oxygenation and hemodynamic parameters deep in the living rodent brain ${ }^{11,17}$, thus enabling direct investigations into mechanisms of neurovascular coupling ${ }^{31}$. Calcium transients and hemodynamic responses occur at significantly different time scales and hence can be distinguished using high-resolution temporal as well as auxiliary multi-spectral OA information (Supp. Fig. 8). Moreover, the capacity to potentially distinguish brain-wide time profiles associated with specific GECIs, further empowers OA with unique capabilities for assessing normal and dysregulated neural network interactions as well as for investigating the roles of other CNS cell types. Future work therefore includes implementation of the common neural network analysis strategies employed in mesoscopic fluorescence imaging and other functional neuroimaging techniques, such as resting-state networks, dynamic causal modelling and stimulus encoding-decoding analyses ${ }^{32}$.

The imaging system was designed to capture neural dynamics in real time as true 3D information across effective fields of view of $\sim 2 \mathrm{~cm}^{3}$ covering an entire mouse brain at a spatial resolution of $150 \mu \mathrm{m}$. In contrast, imaging a whole rodent brain with an advanced optical microscopy technique like OCT still requires slicing it to $\sim 200 \mu \mathrm{m}$ thick layers ${ }^{33}$. To demonstrate this capability, we first tracked whole-brain calcium dynamics in a novel bloodfree isolated-brain preparation. The isolated whole-brain has clear advantages for studying large-scale recording capacity over conventional in vitro preparations like neural cultures ${ }^{2}$ or brain slices ${ }^{34}$, realistically capturing both long-range, whole-brain neuronal network interactions and the brain's light-scattering properties. Multiple aspects of our method can be further improved. Imaging scalability can readily be achieved using high-frequency matrix array probes for better spatial resolution, but at the expense of a smaller FOV ${ }^{11}$, and of increased skull-mediated distortions of the high-frequency ultrasound wave components ${ }^{35}$. In the current implementation, the mouse's head is fixed during the acquisition to prevent motion. Ultrasound arrays with a relatively low number of elements attached to a rodent's head in order to acquire ultrasound echoes and OA signals ${ }^{13,36}$ have recently been designed. Thus, our proposed technique can potentially be adapted to study freely behaving animals. GCaMP6f, the fastest GCaMP6-family indicator ${ }^{30}$, was chosen here since the system's rapid acquisition time $(10 \mathrm{~ms})$ dictated that the indicator dynamics limit the effective temporal resolution. Slower indicator variants can be imaged instead for improved signal characteristics, while other major advances can arise from the development and use of new activity indicators by leveraging the wide tunability of the lasers used in our technology. Other options include the newer generations of GCaMP-type 37,38 and redshifted probes like RGECO ${ }^{39}$, although their peak extinction still falls within the range of strong absorption by hemoglobin. The long-anticipated development of far-red and nearinfrared calcium indicators with absorption peak above $650 \mathrm{~nm}$ would enable OA imaging in the presence of a significantly lower blood absorption background, reaching deeper brain regions in vivo thus potentially enabling non-invasive monitoring of activity across the entire living rodent brain (Fig. 3D). 


\section{METHODS}

\section{Isolated Brain Preparation}

Brains of female CD-1 as well as GCaMP6f-expressing mice aged 8 to 12 weeks were used in these experiments. This animal experiment was carried out in full compliance with the institutional guidelines of the Institute for Biological and Medical Imaging along with approval from the Government District of Upper Bavaria. Mice were injected intraperitoneally with a lethal dose of Ketamine/Xylazine. Subsequently, intracardiac perfusion with ice-cold PBS was performed in order to remove blood from the brain. For this, once the animal was completely anaesthetized, as determined by the absence of a toepinch reflex, surgery began with an incision from the mid abdomen to the sternum. The ventral part of the rib cage was removed to allow access to the heart. The intracardiac perfusion was then carried out after inserting a $25 \mathrm{G}$ butterfly needle into the left ventricle of the heart followed by an incision into the right atrium. The heart was perfused until both liver and lungs turned white, at which stage decapitation was performed. Next, all tissue and skin surrounding the skull was removed and the skull was rinsed with ice-cold PBS to remove any remaining debris. A cut was then made between the skull and the first cervical vertebra exposing the brain stem. Using bone scissors, a second cut was made on both sides of the skull, which extended from the foramen magnum to the external auditory meatus, and then from the molar process up to the lachrymal dorsal aspect of the skull (Suppl. Fig. 1B). Using forceps, the upper skull plate together with the brain was separated from the lower skull and placed into a Petri dish filled with ice-cold, oxygenated artificial cerebrospinal fluid (ACSF). The brain was then carefully separated from the skull using forceps and placed in a second petri dish filled with fresh ACSF. Any remaining hair, debris and blood vessels were removed using fine forceps and a pipette.

\section{Dextran tracing}

Axonal tracer transport requires intact, functioning neurons and dextran-amines coupled to fluorescent molecules are known for being transported in both the anterograde and retrograde direction ${ }^{40} .10 \mathrm{kDa}$ dextran coupled to Texas-red (ThermoFisher Scientific Inc., Waltham, MA, USA) was injected directly into the cortex of the excised brains preparations in order to validate the functionality of axonal transport. The experimental setup is illustrated in Suppl. Fig. 2A. Freshly excised brains were placed in a custom-made chamber filled with ACSF and with a constant supply of carbogen $\left(95 \% \mathrm{O}_{2}, 5 \% \mathrm{CO}_{2}\right)$ to keep the solution oxygenized and the $\mathrm{pH}$ stable at physiological conditions. For intra-brain injection at a depth of $\sim 1 \mathrm{~mm}$ inside the cortex, a wireless robotic injection system (Neurostar, Tuebingen, Germany) using a 15 to $25 \mu \mathrm{m}$ diameter glass microcapillary was utilized. As controls, either PBS or Texas red (without Dextran) were injected under the same conditions. Afterwards, the brains were fixated in paraformaldehyde either immediately after injection, or after being kept in oxygenized ACSF at $4^{\circ} \mathrm{C}$ in the dark for $1 \mathrm{~h}$. Fixated brains were then sliced into $50 \mu \mathrm{m}$-thick sections to evaluate axonal transport. For this, the brains were first dehydrated in a solution of $30 \%$ sucrose at $4^{\circ} \mathrm{C}$ for $48 \mathrm{~h}$ to remove water and prevent icecrystal formation during cryo-slicing. Subsequently, the brains were embedded in optimal cutting temperature compound (Tissue-Tek ${ }^{\circledR}$ O.C.T. compound, VWR, Darmstadt, Germany) and sliced into $50 \mu \mathrm{m}$-thick sections along the coronal plane using a CM 1950 
cryo-slicer (Leica Biosystems, Wetzlar, Germany). These cryo-sections were mounted onto microscope slides and air-dried for $20 \mathrm{~min}$ in the dark. A coverslip was placed on top of the slices and sealed with Vectashield containing DAPI (Vector Laboratories Inc, Burlingame, CA, USA). DAPI stains the DNA and RNA of cells, hence outlining cellular anatomy. Compound brain slice images were captured using an Imager.M2 microscope fitted with shift-free DAPI and Texas red filter sets (Carl Zeiss AG, Oberkochen, Germany). Image acquisition was done using the Zen 2 microscope software (Carl Zeiss AG, Oberkochen, Germany).

\section{Electroencephalography recording}

The experimental setup for electroencephalography (EEG) recordings from the isolated brains is depicted in Suppl. Fig. 2B. The excised brains were placed in the custom-made chamber filled with oxygenized ACSF at room temperature (RT). To induce neuronal activity, $5 \mu \mathrm{L}$ of pentylenetetrazol (PTZ; $100 \mathrm{mg} / \mathrm{ml}$ in PBS) was directly injected into the cortex using a glass capillary and a robotic injection system (Neurostar, Tuebingen, Germany). EEG-signals were recorded via two custom-made needle electrodes, connected to a DP-311 differential amplifier (Warner Instruments, LLC, Hamden, CT, USA). The amplified signals were digitized by means of a PowerLab26T data acquisition module (AD Instruments, Sydney, Australia) controlled through a host PC running the Labchart 8 software (AD Instruments, Sydney, Australia). The amplifier gain value was set to 100 due to relatively low signal strengths for EEG-recordings in isolated brains. For comparison, in vivo EEG of a CD-1 mouse was also recorded, as described previously ${ }^{41}$. This animal experiment was performed in full compliance with the institutional guidelines of the Institute for Biological and Medical Imaging and with approval from the Government District of Upper Bavaria. In order to induce widespread brain activity, the epileptic drug PTZ was injected intraperitoneally ${ }^{42}$. For in vivo EEG-recording, the amplifier was set to a high pass of $10 \mathrm{~Hz}$, a low pass of $100 \mathrm{~Hz}$ and a gain of 100 . After baseline recording, $100 \mu \mathrm{L}$ of PTZ $(25 \mathrm{mg} / \mathrm{ml}$ in PBS) were injected intraperitoneally and EEG-signals were recorded for at least 25 minutes. At the end of the experiment, the mouse was euthanized while still under anesthesia. The Recorded EEG signals were processed using MatLab (MathWorks, Natick, United States) to identify periods of neuronal activity in the brain. For this, the spectrograms of the EEG signals were calculated as the short-time Fourier transform with a window of 20 seconds, which is sufficient to detect higher frequency components in the range of $10-20 \mathrm{~Hz}$ corresponding to seizure-like activity caused by PTZ.

\section{In vivo animal handling}

Eight- to twelve-week-old female C57BL/6J-Tg(Thy1-GCaMP6f)GP5.5Dkim/J and C57BL/6J-Tg(Thy1-GCaMP6s)GP4.12Dkim/J (The Jackson Laboratory, Bar Harbor, ME, USA; stock numbers 024276 and 025776 , respectively) along with six- to twelve-week-old athymic female nude mice (Envigo, New Jersey, USA; stock number Foxn $1^{n u}$ 069) were used for the in vivo experimentation in full compliance with the institutional guidelines of the Institute for Biological and Medical Imaging and with approval from the Government District of Upper Bavaria. Mice were anesthetized with isoflurane $(2.5 \% \mathrm{v} / \mathrm{v}$ for induction) in $100 \% \mathrm{O}_{2}$, and when necessary, any fur on the head of the mouse was removed using consumer hair-removal cream prior to experiments. To avoid motion artifacts during 
imaging, the head of the mouse was fixed into a custom designed stereotactic mouse head holder, which was coupled to an anesthetic breathing mask (Narishige International Limited, London, United Kingdom). During experiments the animals were maintained under isoflurane anesthesia with $1.0 \%$ to $1.5 \% \mathrm{v} / \mathrm{v}$ in $100 \% \mathrm{O}_{2}$ with a flow rate of $\sim 0.7 \mathrm{~L} / \mathrm{min}$ and the physiological parameters (blood oxygenation, heart rate, and body temperature) of the animals were continuously monitored using a PhysioSuite ${ }^{\mathrm{TM}}$ physiological monitor (Kent Scientific, Torrington, Connecticut). The core body temperature was tracked and maintained at $\sim 36^{\circ} \mathrm{C}$ using a homeothermic temperature controller coupled to a heating pad, both being controlled by the PhysioSuite ${ }^{\mathrm{TM}}$. For analgesia, a single drop of $125 \mathrm{mg} / \mathrm{mL}$ metamizole was given orally prior to experiments.

\section{Hindpaw Electrical Stimulation}

For GCaMP imaging hindpaw stimulation was applied by inserting thin stainless steel needle electrodes under the skin of the paw in isoflurane-anesthetized mice. Electrical stimulation with 50 ms-duration pulses at a constant current of $1 \mathrm{~mA}$ was employed using a constant current stimulus isolator (Model A365R, World Precision Instruments, Sarasota, FL, USA). The block stimulation paradigm consisted of repeating the pulses every 20 seconds (Fig. 4C). For the induction and imaging of hemodynamic responses, the following

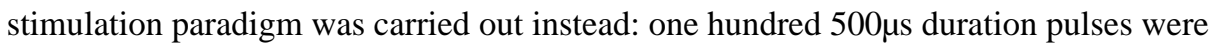
applied at $0.5 \mathrm{~mA}$ every $200 \mathrm{~ms}$ for $20 \mathrm{~s}$. Stimulation began 30 s into the imaging cycle to allow baseline activity approximation. The optoacoustic (OA) and/or fluorescence data acquisitions were synchronized with the start of the first cycle.

\section{Imaging set-up}

The OA tomography system used for real-time volumetric acquisition of data consists of a custom-made spherical transducer array (Imasonic SaS, Voray, France). The spherical surface has a $40 \mathrm{~mm}$ radius and consists of 512 piezocomposite elements covering an angle of $140^{\circ}$ ( $1.32 €$ solid angle). The elements have a diameter of $2.5 \mathrm{~mm}, 5 \mathrm{MHz}$ central frequency and approximately $100 \%-6 \mathrm{~dB}$ detection bandwidth, providing a nearly isotropic resolution of $150 \mu \mathrm{m}$ around the center of the spherical geometry and an effective field of view of approximately $2 \mathrm{~cm}^{3}$. The imaging resolution and field of view were characterized with an agar phantom containing sparsely distributed $\sim 50 \mu \mathrm{m}$ diameter polyethylene absorbing spheres (Cospheric LLC, Santa Barbara, USA). The image of the phantom (top maximum intensity projection) rendered with the system is displayed in Suppl. Fig. 4. The resolution at the center of the spherical array was estimated as the mean square difference between the measured sphere's full width at half maximum and its actual diameter. The field of view was estimated as the size of the region containing particles reconstructed with an amplitude higher than $50 \%$ of the maximum signal in the images.

\section{Isolated brain imaging}

For imaging of the isolated brains, the array was held pointing upwards by a custom-made 3D-printed holder attached to a X-Y positioning platform. The holder further allowed for superfusion of ACSF around the excised brain via an inlet and an outlet, thus establishing a physiological environment supporting brain vitality (Fig. 1A). Excised brains were placed at approximately the center of the spherical geometry lying upon a $\sim 10 \mu \mathrm{m}$ thin transparent 
polyethylene foil. Illumination was provided via a self-made fiber bundle consisting of seven fibers, each with a core diameter of $600 \mu \mathrm{m}$. One of the fibers was inserted into a cylindrical cavity of the spherical array to illuminate the bottom part of the brain while the other 6 fibers were inserted into the holder and equally spaced at $120^{\circ}$ in azimuthal direction and with polar angles of $5.7^{\circ}$ and $37^{\circ}$ (Fig. 1A). The illumination source was an optical parametric oscillator (OPO)-based laser (Innolas $\mathrm{GmbH}$, Krailling, Germany) providing short ( $<10 \mathrm{~ns}$ ) pulses at repetition frequency of $100 \mathrm{~Hz}$ whose optical wavelength is freely tunable between 420 to $680 \mathrm{~nm}$. The $512 \mathrm{OA}$ signals detected by the matrix array elements were simultaneously digitized with a custom-made data acquisition system (Falkenstein Microsysteme GmbH, Taufkirchen, Germany) triggered with the Q-switch output of the laser. The digitized signals were transferred to a computer via $1 \mathrm{Gbit} / \mathrm{s}$ Ethernet connection. To record fluorescence signals, a high speed sCMOS camera (Andor Technology Ltd, Belfast, UK) was positioned on top of the holder pointing downwards and synchronized with the pulsed laser source. The camera was equipped with a manually focusing $105 \mathrm{~mm}$ Nikon F mount objective (Nikon, Chiyoda, Tokio, Japan) and a one-inch bandpass filter (525 nm center wavelength and $39 \mathrm{~nm}$ bandwidth; MF525-39, Thorlabs Inc, Newton, USA). The acquisition time of the camera was set to $160 \mathrm{~ms}$, corresponding to the integration of 4 laser pulses.

\section{In vivo imaging experiments}

For the in vivo imaging experiments, the OA tomography system was used in a reversed orientation (Suppl. Fig. 6B) The wide-field fluorescence recordings were not performed concurrently due to the lack of access to the imaged area in the in vivo setting. Also, the laser light beam was guided differently from the isolated brain setup, i.e. a single custommade silica fused-end fiber bundle (CeramOptics GmbH, Bonn, Germany) was used to broadly illuminate the imaged area from a single direction. At the peak absorption wavelength of calcium-saturated GCaMP $6 \mathrm{f} / 6 \mathrm{~s}$ of $488 \mathrm{~nm}$, the light fluence at the sample was measured to be $\sim 3 \mathrm{~mJ} / \mathrm{cm}^{2}$, i.e. below safe exposure limits for pulsed laser radiation ${ }^{43}$. The same fiber bundle was used to illuminate the shaved mouse head. However, fluorescence imaging was performed separately with the mouse head illuminated laterally to avoid blinding the camera, as indicated in Suppl. Fig. 6A.

Imaging of GCaMP-responses were done at $25 \mathrm{~Hz}$ laser repetition frequency and a single excitation wavelength of $488 \mathrm{~nm}$. Optoacoustic imaging of hemodynamic imaging was carried out instead at $20 \mathrm{~Hz}$ with five wavelengths $(530,540,560,575$ and $585 \mathrm{~nm})$. This resulted in an imaging rate of $4 \mathrm{~Hz}$ per wavelength and allowed for the unmixing of two blood components: oxygenated hemoglobin ( $\mathrm{HbO}$ ) and reduced hemoglobin ( $\mathrm{HbR})$. Total Hemoglobin (HbT) was estimated as the sum of these two components. A $100 \mu \mathrm{m}$ black microsphere (BKPMS-1.2 90-106um, Cospheric, USA) was placed in the field of view, right above the skin of the mouse and used to normalize the optoacoustic images with the per-pulse laser energy variations (assumed to be proportional to the optoacoustic signal of the sphere). 


\section{Isolated protein measurements}

The optoacoustic spectrum of the isolated proteins was measured with the same system by injecting the proteins $(\sim 50 \mu \mathrm{M}$ concentration) in a polyethylene tubing with $0.57 \mathrm{~mm}$ inner diameter. The laser was tuned between 420 and $580 \mathrm{~nm}$ with $5 \mathrm{~nm}$ step on a per pulse basis and averaging of 50 multi-spectral datasets was performed. For the measurements, the proteins were first diluted in a buffer containing $30 \mathrm{mM} 4$-Morpholinepropanesulfonic acid (MOPS), $100 \mathrm{mM} \mathrm{KCl}$ (pH 7.2) and $1 \mathrm{mM} \mathrm{MgCl}_{2}$ was subsequently added to mimic physiological conditions. Calcium-free and calcium-saturated protein-solutions were rendered by adding $10 \mathrm{mM}$ EGTA orn $100 \mu \mathrm{M} \mathrm{CaCl}_{2}$, respectively, to the solution.

\section{Imaging data analysis and processing}

Volumetric (3D) OA images were reconstructed from the acquired signals with a graphics processing unit (GPU)-based implementation of a back-projection formula ${ }^{44}$. Prior to reconstruction, the signals were deconvolved with the impulse response of the transducer array elements and band-pass filtered with cut-off frequencies of 0.1 and $6 \mathrm{MHz}$.

Reconstruction was performed on a grid of $150 \times 150 \times 100$ voxels $^{3}\left(15 \times 15 \times 10 \mathrm{~mm}^{3}\right)$ to better match the spatial resolution of the system. The reconstructed images were normalized with the estimated light fluence distribution $\Phi(\vec{r}, t)$ to compensate for light attenuation within the sample according to the light diffusion equation with no transient effects and no internal light sources ${ }^{45}$

$$
\mu_{a} \Phi(\vec{r}, t)-D \nabla^{2} \Phi(\vec{r}, t)=0
$$

where $\mu_{a}$ is the optical absorption coefficient and $D=1 / 3\left(\mu_{a}+\mu^{\prime}{ }_{s}\right)$, being $\mu^{\prime}{ }_{s}$ the reduced scattering coefficient. The solution of Eq. 1 for a spherical uniform sample uniformly illuminated on its surface was considered as an estimation of the light fluence distribution, which corresponds to a modified spherical Bessel function given by 46

$$
\Phi(r, t)=\frac{\Phi_{0}}{\frac{\sinh (k R)}{k R}} \frac{\sinh (k r)}{k r}
$$

where $R$ is the radius of the sphere, $r$ is the distance from its center, $\Phi_{0}$ is the fluence at the surface and $k=\sqrt{\mu_{a} / D}$. A predictive Kalman filter was further applied on a per-slice basis to the time domain of the sequence of reconstructed OA images for noise removal ${ }^{47}$.

The effect of bleaching in OA signal decay during the stimulation-evoked activity experiment was estimated as follows. A Kalman filter with gain 0.75 was applied to the individual time profiles of the OA signal intensity for a location in the brain having a clear activation peak. The average time profile for all cycles was taken as reference. All profiles were windowed to the initial $4 \mathrm{~s}$ following the hind paw stimuli. The OA signal intensity associated to GCaMP as a function of time was estimated by cross-correlation of the individual profiles and the reference profile. A 10-point moving average filter was applied to the calculated cross-correlation values, which were subsequently normalized to the maximum value. An exponential decay function was eventually fitted to these values. 
The onset time for the appearance of activity after PTZ injection in the isolated brain experiments was estimated as follows. First, the standard deviation of the background OA signal intensity for each voxel of the reconstructed image was calculated for a window of 300 consecutive frames (40ms per frame) where no PTZ-related activity occurred. The onset time was estimated by considering the time point at which the OA signal exceeds the standard deviation of the background signal by a factor of 3 . It was assumed that no activation was produced at voxels for which the OA signal intensity did not reach this value.

For analysis of the hemodynamics-related optoacoustic signal changes, the multi-spectral optoacoustic data was first reconstructed as outlined above on a per-wavelength basis. A spatial moving average of $3 \times 3$ voxel and a temporal forward moving average of 10 frames (2.5s) was applied to the time-lapse volumetric data sets. The images were then unmixed for $\mathrm{HbO}, \mathrm{HbR}$ and $\mathrm{HbT}$ on a per-voxel basis according to their absorption spectrum at the five excitation wavelengths by assuming a linear mixing model ${ }^{17,41}$. Delta calculations were performed for each component using the first 9 frames before stimulation as a baseline estimation. Lastly a low pass filter with a cutoff frequency of $0.05 \mathrm{~Hz}$ was used to smoothen the resulting temporal profiles.

\section{Supplementary Material}

Refer to Web version on PubMed Central for supplementary material.

\section{ACKNOWLEDGEMENTS}

The authors acknowledge grant support from the European Research Council (under grant agreement ERC-2015CoG-682379) and the US National Institutes of Health (grants R21-EY026382 and UF1-NS107680). We also acknowledge the help of $\mathrm{N}$. Tritsch and L. Mcley with reading and commenting on the manuscript.

\section{REFERENCES}

1. Hilgetag CC \& Amunts K Connectivity and cortical architecture. e-Neuroforum 7, 56-63, doi: 10.1007/s13295-016-0028-0 (2016).

2. Peron S, Chen TW \& Svoboda K Comprehensive imaging of cortical networks. Current opinion in neurobiology 32, 115-123, doi:10.1016/j.conb.2015.03.016 (2015). [PubMed: 25880117]

3. Eggebrecht AT et al. Mapping distributed brain function and networks with diffuse optical tomography. Nat Photonics 8, 448-454, doi:10.1038/nphoton.2014.107 (2014). [PubMed: 25083161]

4. Errico $\mathrm{C}$ et al. Transcranial functional ultrasound imaging of the brain using microbubble-enhanced ultrasensitive Doppler. Neuroimage 124, 752-761, doi:10.1016/j.neuroimage.2015.09.037 (2016). [PubMed: 26416649]

5. Schulz K et al. Simultaneous BOLD fMRI and fiber-optic calcium recording in rat neocortex. Nature methods 9, 597-602, doi:10.1038/nmeth.2013 (2012). [PubMed: 22561989]

6. Looger LL \& Griesbeck O Genetically encoded neural activity indicators. Current opinion in neurobiology 22, 18-23, doi:10.1016/j.conb.2011.10.024 (2012). [PubMed: 22104761]

7. Yang W \& Yuste R In vivo imaging of neural activity. Nature Meth 14, 349-359, doi:10.1038/ nmeth.4230 (2017).

8. Bouchard MB et al. Swept confocally-aligned planar excitation (SCAPE) microscopy for high speed volumetric imaging of behaving organisms. Nature Phot 9, 113-119, doi:10.1038/nphoton.2014.323 (2015).

9. Dana $\mathrm{H}$ et al. Thy1-GCaMP6 transgenic mice for neuronal population imaging in vivo. PLoS One 9, e108697, doi:10.1371/journal.pone.0108697 (2014). [PubMed: 25250714] 
10. Prevedel R et al. Fast volumetric calcium imaging across multiple cortical layers using sculpted light. Nature Meth 13, 1021-1028, doi:10.1038/nmeth.4040 (2016).

11. Dean-Ben XL et al. Functional optoacoustic neuro-tomography for scalable whole-brain monitoring of calcium indicators. Light Sci Appl. 5, e16201, doi:10.1038/1sa.2016.201 (2016). [PubMed: 30167137]

12. Gottschalk S, Fehm TF, Dean-Ben XL, Tsytsarev V \& Razansky D Correlation between volumetric oxygenation responses and electrophysiology identifies deep thalamocortical activity during epileptic seizures. Neurophotonics 4, 011007, doi:10.1117/1.NPh.4.1.011007 (2017). [PubMed: 27725948]

13. Tang J, Coleman JE, Dai X \& Jiang H Wearable 3-D Photoacoustic Tomography for Functional Brain Imaging in Behaving Rats. Scientific reports 6, 25470, doi:10.1038/srep25470 (2016). [PubMed: 27146026]

14. Dean-Ben XL, Gottschalk S, Sela G, Shoham S \& Razansky D Functional optoacoustic neurotomography of calcium fluxes in adult zebrafish brain in vivo. Optics Lett 42, 959-962, doi: 10.1364/OL.42.000959 (2017).

15. Wang LV \& Yao J A practical guide to photoacoustic tomography in the life sciences. Nature methods 13, 627-638, doi:10.1038/nmeth.3925 (2016). [PubMed: 27467726]

16. Ermolayev V, Dean-Ben XL, Mandal S, Ntziachristos V \& Razansky D Simultaneous visualization of tumour oxygenation, neovascularization and contrast agent perfusion by real-time threedimensional optoacoustic tomography. Eur Radiol 26, 1843-1851, doi:10.1007/ s00330-015-3980-0 (2016). [PubMed: 26334513]

17. Gottschalk S, Fehm TF, Dean-Ben XL \& Razansky D Noninvasive real-time visualization of multiple cerebral hemodynamic parameters in whole mouse brains using five-dimensional optoacoustic tomography. J Cereb Blood Flow Metab 35, 531-535, doi:10.1038/jcbfm.2014.249 (2015). [PubMed: 25586142]

18. Knieling F et al. Multispectral Optoacoustic Tomography for Assessment of Crohn's Disease Activity. New Engl J Med 376, 1292-1294, doi:10.1056/NEJMc1612455 (2017). [PubMed: 28355498]

19. Tzoumas $\mathrm{S}$ et al. Eigenspectra optoacoustic tomography achieves quantitative blood oxygenation imaging deep in tissues. Nat Commun 7, 12121, doi:10.1038/ncomms12121 (2016). [PubMed: 27358000]

20. Yao J et al. High-speed label-free functional photoacoustic microscopy of mouse brain in action. Nature methods 12, 407-410, doi:10.1038/nmeth.3336 (2015). [PubMed: 25822799]

21. Schmued L, Kyriakidis K \& Heimer L In vivo anterograde and retrograde axonal transport of the fluorescent rhodamine-dextran-amine, Fluoro-Ruby, within the CNS. Brain research 526, 127-134 (1990). [PubMed: 1706635]

22. Velíšek 1. Models of Generalized Seizures in Freely Moving Animals. In book: Reference Module in Neuroscience and Biobehavioral Psychology. 2017, doi: 10.1016/B978-0-12-809324-5.00129-2.

23. Dhir A Pentylenetetrazol (PTZ) kindling model of epilepsy. Curr Protoc Neurosci Chapter 9, Unit9 37, doi:10.1002/0471142301.ns0937s58 (2012).

24. Tang J et al. Noninvasive high-speed photoacoustic tomography of cerebral hemodynamics in awake-moving rats. J Cereb Blood Flow Metab 35, 1224-1232, doi:10.1038/jcbfm.2015.138 (2015). [PubMed: 26082016]

25. Gottschalk $\mathrm{S}$ et al. Short and long-term phototoxicity in cells expressing genetic reporters under nanosecond laser exposure. Biomaterials 69, 38-44, doi:10.1016/j.biomaterials.2015.07.051 (2015). [PubMed: 26280948]

26. Norup Nielsen A \& Lauritzen M Coupling and uncoupling of activity-dependent increases of neuronal activity and blood flow in rat somatosensory cortex. The Journal of physiology 533, 773785 (2001). [PubMed: 11410634]

27. Kozberg MG, Ma Y, Shaik MA, Kim SH \& Hillman EM Rapid Postnatal Expansion of Neural Networks Occurs in an Environment of Altered Neurovascular and Neurometabolic Coupling. J Neurosci 36, 6704-6717, doi:10.1523/JNEUROSCI.2363-15.2016 (2016). [PubMed: 27335402] 
28. Vanni MP \& Murphy TH Mesoscale transcranial spontaneous activity mapping in GCaMP3 transgenic mice reveals extensive reciprocal connections between areas of somatomotor cortex. $\mathrm{J}$ Neurosci 34, 15931-15946, doi:10.1523/JNEUROSCI.1818-14.2014 (2014). [PubMed: 25429135]

29. Schroeter A, Grandjean J, Schlegel F, Saab BJ, \& Rudin M Contributions of structural connectivity and cerebrovascular parameters to functional magnetic resonance imaging signals in mice at rest and during sensory paw stimulation. J Cereb Blood Flow Metab. 37, 2368-2382 (2017). [PubMed: 27596833]

30. Chen T-W, Wardill TJ, Sun Y, Pulver SR, Renninger SL, Baohan A, Schreiter ER, Kerr RA, Orger MB, Jayaraman V, Looger LL, Svoboda K \& Kim DS Ultrasensitive fluorescent proteins for imaging neuronal activity. Nature 499, 295-300 (2013) [PubMed: 23868258]

31. O'Herron P et al. Neural correlates of single-vessel haemodynamic responses in vivo. Nature 534, 378-382, doi:10.1038/nature17965 (2016). [PubMed: 27281215]

32. Razi A \& Friston KJ The connected brain: causality, models, and intrinsic dynamics IEEE Signal Process. Mag 33, 14-35 (2016).

33. Lefebvre J, Castonguay A, Pouliot P, Descoteaux M \& Lesage F Whole mouse brain imaging using optical coherence tomography: reconstruction, normalization, segmentation, and comparison with diffusion MRI. Neurophotonics 4, 041501, doi:10.1117/1.NPh.4.4.041501 (2017). [PubMed: 28721357]

34. Llinás RR, Leznik E \& Urbano FJ Temporal binding via cortical coincidence detection of specific and nonspecific thalamocortical inputs: a voltage-dependent dye-imaging study in mouse brain slices. Proceedings of the National Academy of Sciences 99, 449-454 (2002).

35. Kneipp M et al. Effects of the murine skull in optoacoustic brain microscopy. Journal of biophotonics 9, 117-123, doi:10.1002/jbio.201400152 (2016). [PubMed: 25919801]

36. Sieu LA et al. EEG and functional ultrasound imaging in mobile rats. Nature Meth 12, 831-834, doi:10.1038/nmeth.3506 (2015).

37. Badura A, Sun XR, Giovannucci A, Lynch LA \& Wang SS Fast calcium sensor proteins for monitoring neural activity. Neurophotonics 1, 025008, doi:10.1117/1.NPh.1.2.025008 (2014). [PubMed: 25558464]

38. Dana H et al. High-performance GFP-based calcium indicators for imaging activity in neuronal populations and microcompartments. bioRxiv doi: 10.1101/434589 (2018).

39. Akerboom $J$ et al. Genetically encoded calcium indicators for multi-color neural activity imaging and combination with optogenetics. Front Mol Neurosci 6, 2, doi:10.3389/fnmol.2013.00002 (2013). [PubMed: 23459413]

40. Schmued L, Kyriakidis K, Heimer L, In vivo anterograde and retrograde axonal transport of the fluorescent rhodamine-dextran-amine, Fluoro-Ruby, within the CNS. Brain Res 526, 127-134 (1990). [PubMed: 1706635]

41. Gottschalk S, Fehm TF, Dean-Ben XL, Tsytsarev V, Razansky D, Correlation between volumetric oxygenation responses and electrophysiology identifies deep thalamocortical activity during epileptic seizures. Neurophotonics 4, 011007 (2017). [PubMed: 27725948]

42. Tang J et al., Noninvasive high-speed photoacoustic tomography of cerebral hemodynamics in awake-moving rats. J Cereb Blood Flow Metab 35, 1224-1232 (2015). [PubMed: 26082016]

43. American National Standards for the Safe Use Lasers. ANSI Z136.1. Orlando, FL: American Laser Institute; 2000.

44. Dean-Ben XL, Ozbek A, Razansky D, Volumetric real-time tracking of peripheral human vasculature with GPU-accelerated three-dimensional optoacoustic tomography. IEEE Trans Med Imag 32, 2050-2055 (2013).

45. L. V. a. W. Wang H, Biomedical Optics: Principles and Imaging. (Wiley, New jersey, 2007).

46. Abramowitz M. a. I. A., Handbook of mathematical functions: With formulas, graphs, and mathematical tables., (Courer Corporation., 1964), vol. 55.

47. P. a. M. Zarchan H, Fundamentals of Kalman Filtering: A Practical Approach., (American Institute of Aeronautics and Astronautics, Incorporated., 2000). 

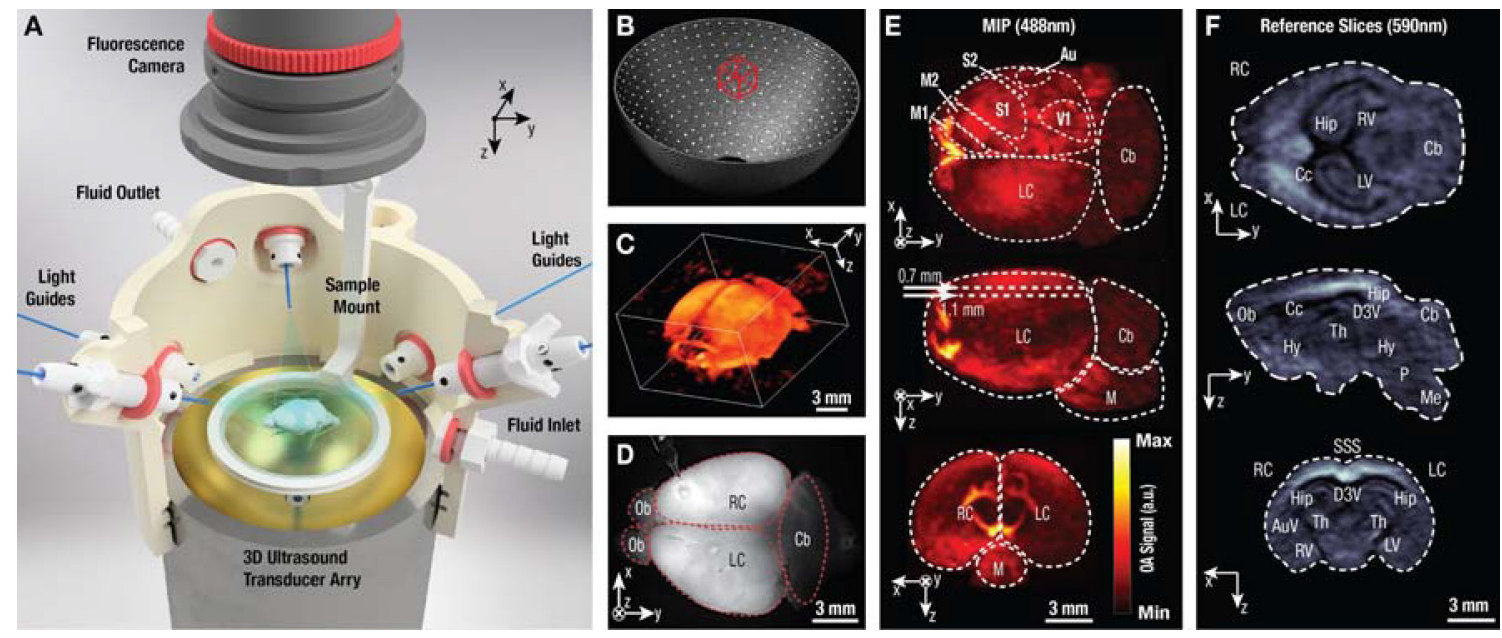

Figure 1.

Bi-modal optoacoustic and fluorescence imaging of isolated brains. (A) 3D-rendering of the imaging setup featuring a 512-element spherical matrix transducer array inserted into a custom-made 3D-printed holder. The holder features an inlet and outlet to facilitate perfusion with ACSF while the excised brain is placed on top of a transparent polyethylene foil that is attached to the submerged sample mount. The brain is evenly illuminated via seven light guides (fibers) and a sensitive sCMOS camera is positioned at the top for concurrent wide-field fluorescence imaging. (B) The distribution of detection elements on the spherical array with its effective volumetric imaging field of view indicated by the red bounding box. (C) Representative volumetric optoacoustic data recorded from an excised GCaMP6f-brain at $488 \mathrm{~nm}$ excitation wavelength. (D) The corresponding planar fluorescence image. The glass capillary visible on top of the right cortex was used for intracortical injections. (E) Maximum intensity projections (axial, sagittal and coronal) of the 3D optoacoustic dataset shown in C. (F) Representative coronal, sagittal and transverse orthoslices (top to bottom) of the 3D optoacoustic image acquired at $590 \mathrm{~nm}$. Au: auditory cortex; AuV: secondary auditory cortex; Cb: cerebellum; Cc: corpus callosum; D3V: dorsal third ventricle; Hip: hippocampus; Hy: hypothalamus; M: medulla; Ob: olfactory bulb; R/LC: right/left cortex; R/LV: right/left ventricle; M1,M2: primary and secondary motor cortex; Me: medulla; Ob: olfactory bulbs; P: pons; S1,S2: primary and secondary somatosensory cortex; Th: thalamus; V1: primary visual cortex. 
A $\quad$ B
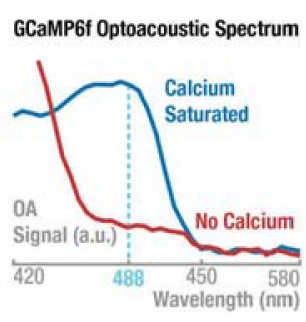

B

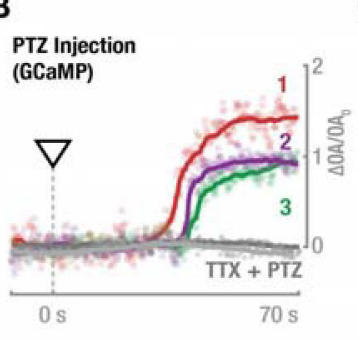

E

E $\quad 23.0 \mathrm{~s} \quad 36.0 \mathrm{~s}$
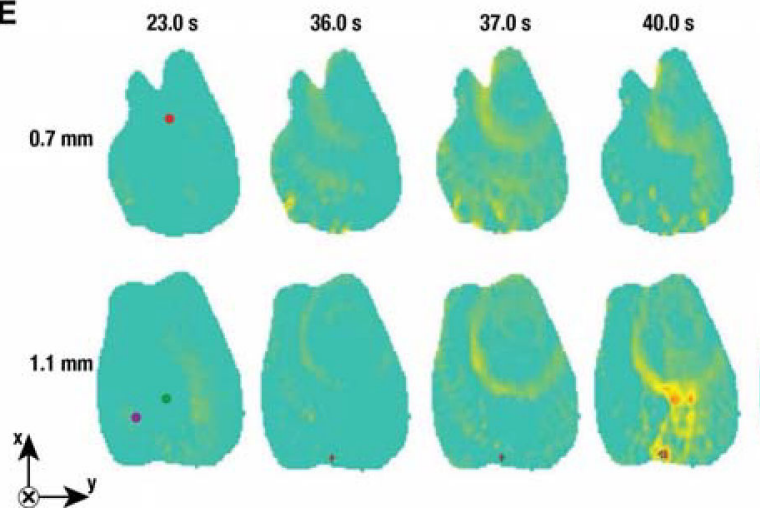
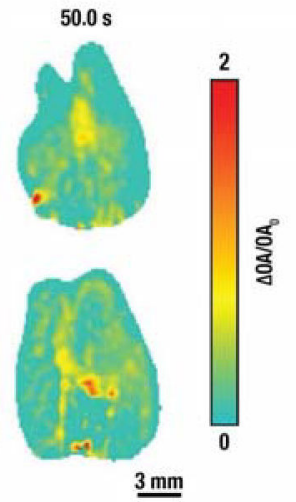

D

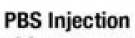

(GCaMP control)

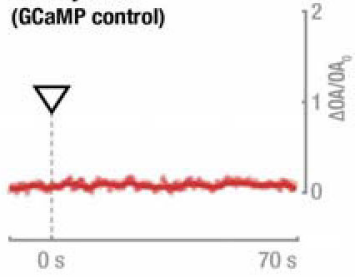

$\mathbf{F}$

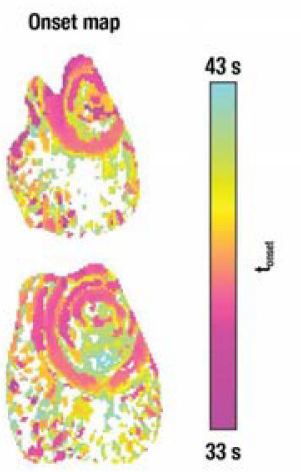

Figure 2.

Whole-brain volumetric optoacoustic imaging of neuronal activation in the isolated brain model. (A) Optoacoustically measured absorption spectrum of purified calcium-saturated (blue) and calcium-free (red) GCaMP6f-proteins. The dotted line indicates maximum difference in optoacoustic signal at $488 \mathrm{~nm}$ between the two protein states. (B) Time-traces of the normalized optoacoustic data along with the moving averages over 50 image frames (solid lines) are shown from individual voxels whose position is indicated in panel E. Notably, the activation first occurs in the red voxel located close to the injection site (see the glass capillary location in Fig. 1D), slowly propagating into the rest of the brain (purple and green voxels). Gray traces: Tetrodotoxin (TTX) was injected 180s prior to PTZ, abolishing the activation. (C) Optoacoustic recordings from a control experiment with an isolated CD-1 mouse brain not expressing GCaMP6f proteins. No changes due to PTZ-injection are detected. (D) Results of an additional control experiment where an isolated GCaMP6fexpressing brain was injected with a phosphate buffered saline, resulting in no optoacoustic signal changes. (E) Temporal evolution of the relative optoacoustic signal changes $(\triangle \mathrm{OA} / \mathrm{OA})$ in two representative slices located at depths of $0.7 \mathrm{~mm}$ and $1.1 \mathrm{~mm}$ in a GCaMP6f-expressing brain (the slice locations are indicated in Fig. 1E). Injection of the PTZ neurostimulant was done at $t=0$ s. (F) Onset activation map for the experiment shown in (A), generated for each image voxel by calculating the time point $\left(t_{\text {onset }}\right)$ when the relative optoacoustic signal change $(\triangle \mathrm{OA} / \mathrm{OA})$ exceeded 3 times the standard deviation of the background signal (before the PTZ injection). The white color represents inactivated voxels. 
A

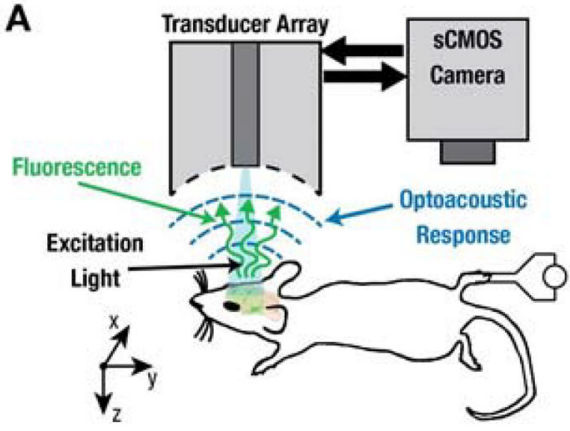

C

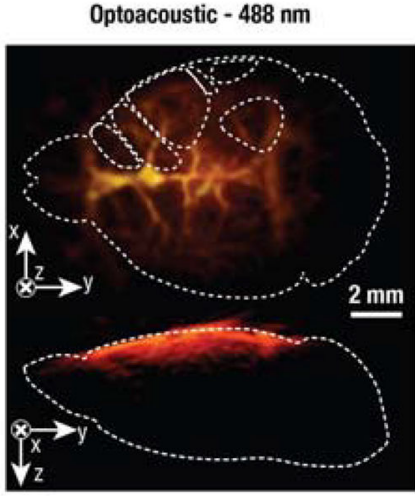

$\mathbf{E}$

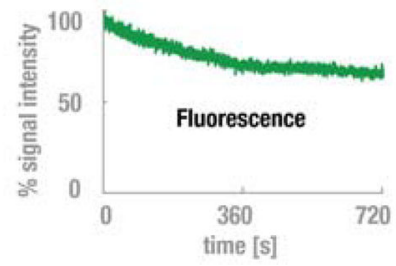

B

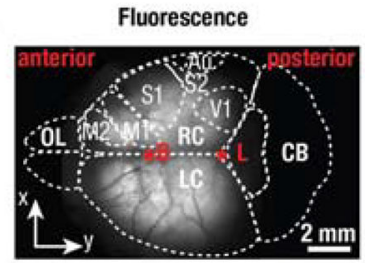

D
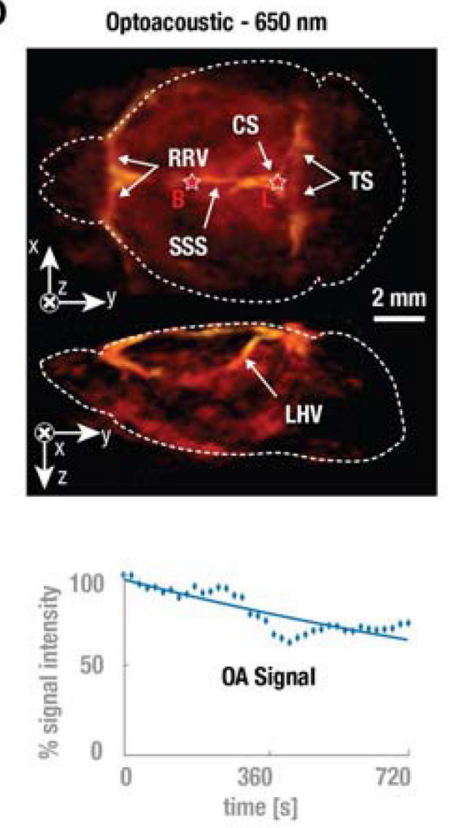

Figure 3.

Non-invasive imaging of the GCaMP6f-brain in vivo. (A) Experimental schematic of hind paw electrical stimulation experiment with alternate imaging in either optoacoustic or fluorescence mode. (B) Representative planar fluorescence image of a GCaMP6f mouse brain. OL: olfactory bulb; LC/RC: left/right cortex; CB: cerebellum; M1,M2: primary and secondary motor cortex; S1,S2: primary and secondary somatosensory cortex; Au: auditory cortex; V1: primary visual cortex. The approximate bregma- and lambda skull-landmarks (red asterisks, B and L) are also indicated. (C) Maximum intensity projections of the representative volumetric optoacoustic image recorded non-invasively from a GCaMP6fexpressing mouse brain at $488 \mathrm{~nm}$. Note the shallow penetration depth of $1-2 \mathrm{~mm}$ at this wavelength. (D) Maximum intensity projections of the volumetric optoacoustic images recorded non-invasively at $650 \mathrm{~nm}$ in vivo, demonstrating the whole-brain recording capacity of the system down to $\sim 7 \mathrm{~mm}$ depth. Bregma- and lambda skull-landmarks are indicated (red asterisks, B and L). Major veins are visible and have been labeled accordingly (RRV: rostral rhinal vein; SSS: superior sagittal sinus; CS: confluence of sinuses; TS: transverse sinus; LHV: longitudinal hippocampal vein). (E) The GCaMP6f-protein is prone to bleaching over the course of the imaging experiment. The normalized average fluorescence signal of the 
whole brain area is plotted (left panel). For optoacoustic (OA) signal decay, the averages of each stimulus cycle (blue diamonds) and a linear fit (blue line) are plotted. 
A

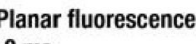

$0 \mathrm{~ms}$
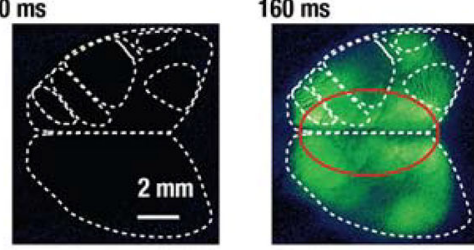

B

Optoacoustic

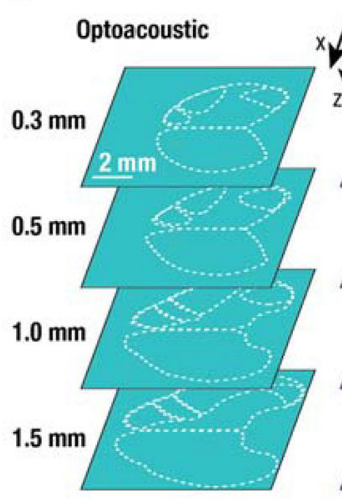

C Electric Stim Train
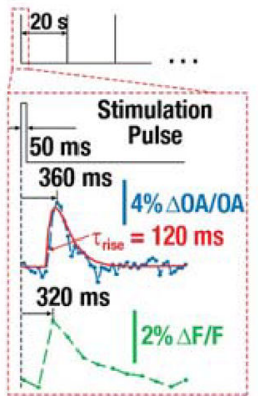

D

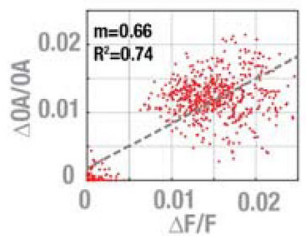

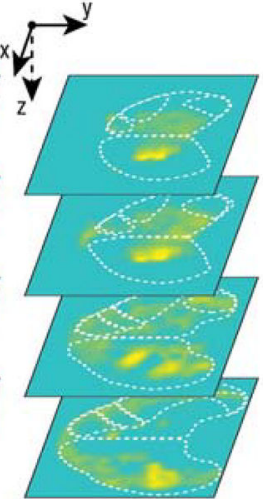

E Fluorescence $2 \% \Delta F / F$ $500 \mathrm{~ms}$

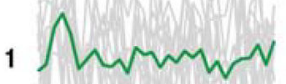

2

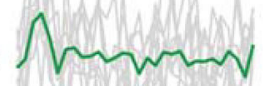

3

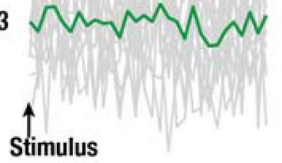

$320 \mathrm{~ms}$
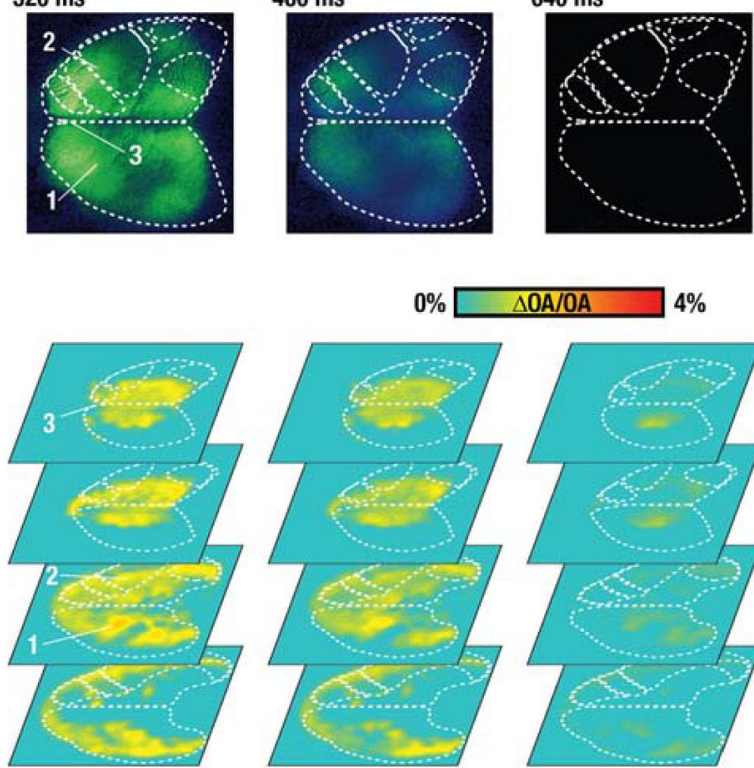

F Optoacoustic

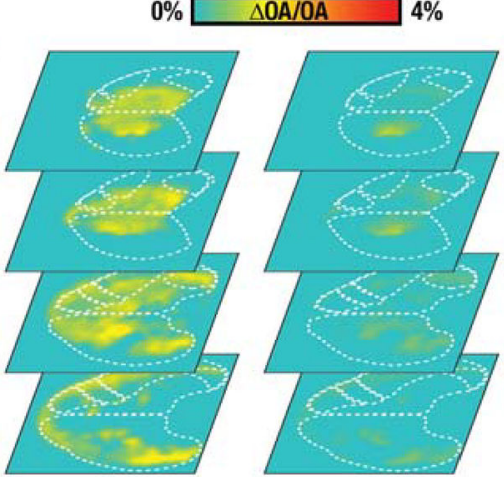

L

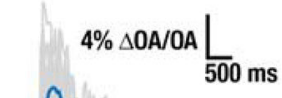

G Optoacoustic GCaMP-negative
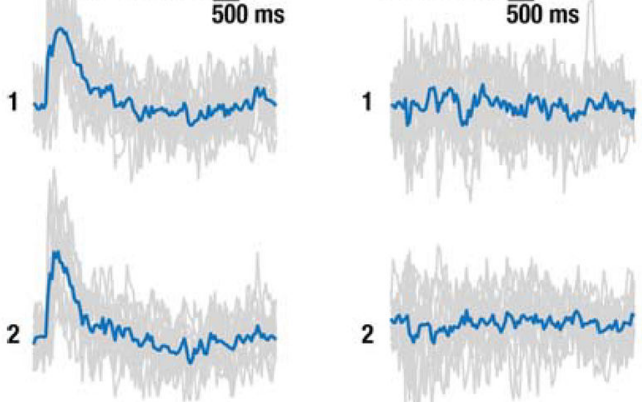

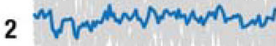
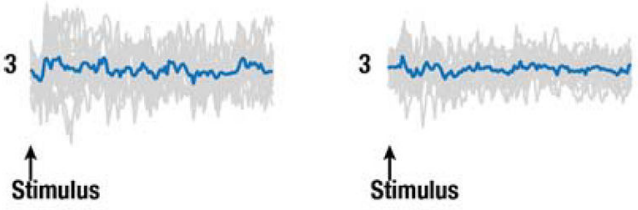

Figure 4.

Non-invasive imaging of somatosensory-evoked rapid calcium transients in the GCaMP6fbrain in vivo. (A) Temporal sequence of fluorescence-recorded brain activation maps $(\Delta \mathrm{F} / \mathrm{F})$ in response to the hindpaw current stimulus at $t=0$. Functional regions of the brain are indicated as dashed white lines. (B) 4D optoacoustically-recorded brain activation maps $(\triangle \mathrm{OA} / \mathrm{OA})$ in response to the stimulus at $t=0$. Slices across the entire brain are shown located at different depths from the brain surface. Functional regions of the brain are indicated as dashed white lines. (C) Schematic of the electrical paw stimulation paradigm. 
One $50 \mathrm{~ms}$ long stimulus was repeated every 20 seconds (top panel). Below, representative single-voxel optoacoustic and single-pixel fluorescence time traces are shown. Note that the volumetric optoacoustic recordings (blue) were carried out at a $25 \mathrm{~Hz}$ rate versus $6.25 \mathrm{~Hz}$ for planar fluorescence (green). (D) Relative optoacoustic signal increase versus relative fluorescence signal increase for a set of points in the region indicated in red in (A) and for three different time points $(160 \mathrm{~ms}, 320 \mathrm{~ms}$ and $640 \mathrm{~ms})$. The maximum of all slices is taken for calculating the relative optoacoustic signal increase. The dashed line of the best fit was determined using a least square method - its slope and coefficient of determination are denoted by $m$ and $\mathrm{R}^{2}$, respectively. (E) Background-subtracted and normalized $(\Delta \mathrm{F} / \mathrm{F})$ fluorescence signal traces following the stimulus in three pixels located in the primary somatosensory cortex on each hemisphere (1 and 2) and inside the superior sagittal sinus (3). (F) Background-subtracted and normalized $(\triangle \mathrm{OA} / \mathrm{OA})$ optoacoustic signal response traces for single voxels located bilaterally in the primary somatosensory cortex outside major vessels (voxels 1 and 2, approx. 1mm depth) and inside the superior sagittal sinus (voxel 3). (G) Background-subtracted and normalized $(\triangle \mathrm{OA} / \mathrm{OA})$ optoacoustic signal traces following the stimulus in control wild-type mice are shown for single voxels at similar locations as in (F). 
A

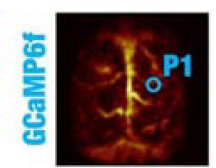

OA (a.u.)

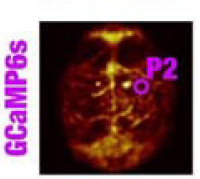

C

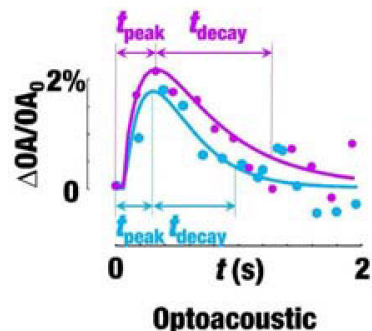

Optoacoustic

B
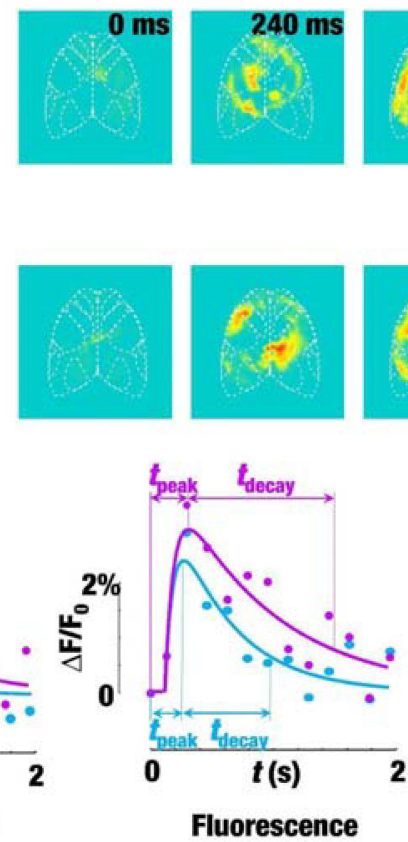

$\mathbf{0} \%$

$\triangle O A / O A$

$2.25 \%$
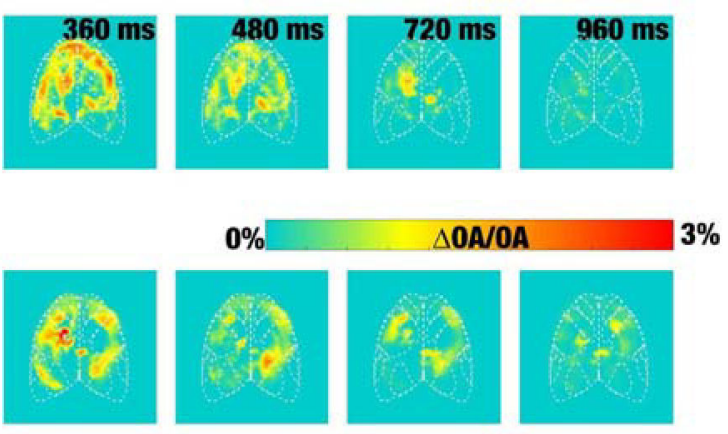

D

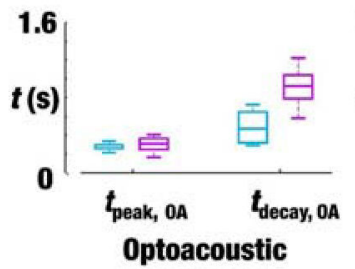

Figure 5.

Comparison of GCaMP6f and GCaMP6s responses to electrical stimulation of the hind paw.

(A) Maximum intensity projection along the depth direction of the three-dimensional images of GCaMP6f- (top) and GCaMP6s-expressing (bottom) mice. (B) Relative increase in optoacoustic signal for a slice at approximately $1 \mathrm{~mm}$ depth at different time points following the stimulation pulse. (C) Fitted time profiles of the relative increase in optoacoustic and fluorescence signals corresponding to locations labeled in (A) for the GCaMP6f (blue) and GCaMP6s (violet) mice. (D) Statistical distribution of the estimated signal time-to-peak $\left(\mathrm{t}_{\text {peak }}\right)$ and decays $\left(\mathrm{t}_{\text {decay }}\right)$ values within the cortex. 